Groups Geom. Dyn. 6 (2012), 83-96

DOI $10.4171 / \mathrm{GGD} / 151$
Groups, Geometry, and Dynamics

(C) European Mathematical Society

\title{
Hyperbolic alternating virtual link groups
}

\author{
Jens Harlander
}

\begin{abstract}
We study the topology and geometry of virtual link complements and groups. We show that the groups defined by the Wirtinger presentation of certain prime dense alternating virtual links are $\mathrm{CAT}(0)$ and hyperbolic.
\end{abstract}

Mathematics Subject Classification (2010). 57M05, 57M50, 20F65, $20 \mathrm{~F} 67$.

Keywords. Alternating virtual knot, hyperbolic group, Wirtinger complex, non-positively curved square complex.

\section{Introduction}

This article is concerned with the geometry and topology of virtual link complements and groups. The notion of a virtual knot appeared first in [11]. Much is known in the classical knot setting. As consequence of Thurston's geometrization theorem for Haken manifolds, all knot groups are fundamental groups of non-positively curved spaces and knot complements are hyperbolic in case the knot is neither a torus nor a satellite knot. A virtual knot is a proper embedding $k: I \rightarrow F \times I$, where $I=[0,1]$ is the unit interval and $F$ is a compact orientable surface with one boundary component, and both $k(0)$ and $k(1)$ are contained in $\partial F \times(0,1)$. If we have more than one properly embedded interval we speak of a virtual link. More generally, one could allow surfaces with none or more than one boundary component and also embedded circles, but we will not do so here. A projection of the virtual link to the top surface $F \times\{1\}$ is a 4-regular graph in $F$ with over- and under-crossing information provided at the vertices. Given a virtual link projection, we can read off a Wirtinger presentation in the usual way. Such Wirtinger presentations are best encoded via labeled oriented graphs. A labeled oriented graph is a finite oriented (not necessarily connected) graph $\Gamma$ on vertices $\{a, b, c, \ldots\}$ and every oriented edge is labeled by a vertex. The associated Wirtinger presentation has generators in one-to-one correspondence with the vertices $\{a, b, c, \ldots\}$, and relations in one-to-one correspondence with the edges. An edge in $\Gamma$ starting at $a$ and ending at $b$, labeled by $c$, gives the relation $a c=c b$ in the Wirtinger presentation. Note that Wirtinger presentations that come from virtual links are encoded by labeled oriented graphs where each connected component of 
the graph is a subdivided interval. The components of the graph are in one-to-one correspondence with the components of the virtual link. A virtual link group is a group defined by the Wirtinger presentation of a virtual link.

Labeled oriented graphs and their Wirtinger presentations arose first in the study of higher dimensional knot theory. The Wirtinger complex, that is the standard 2complex built from a Wirtinger presentation, of a labeled oriented tree is the spine of a ribbon disc complement in the 4-ball. The question of whether Wirtinger complexes of labeled oriented trees are aspherical has been extensively studied because of its well-known relevance to Whitehead's asphericity conjecture: The subcomplex of an aspherical 2-complex is aspherical. Howie showed (see [6], [7]) that if a finite 2-complex $K$ is the subcomplex of a 2-complex that 3-deforms to a point, then $K$ is homotopically equivalent to a Wirtinger complex of a labeled oriented tree. Of course, Wirtinger complexes of classical knots (properly embedded intervals in the 3-ball) are known to be aspherical, but the proof of this fact relies on 3-manifold techniques and no combinatorial proof that would extend to labeled oriented trees has been found. Except in the alternating case (see Weinbaum [18], Wise [19], and [3], p. 220). For surveys of results concerning Whitehead's conjecture see Bogley [4] and Rosebrock [17].

Recall that an alternating knot projection on a sphere is called prime if an embedded disc whose boundary intersects the knot in exactly two points contains all or no crossing in its interior. This can also be expressed in terms of the cell decomposition of the sphere dual to the projection, made up of 4-gons, one for each crossing. The knot projection is prime if every reduced edge loop in the one skeleton of the cell decomposition dual to the projection has length at least four. In analogy one can define a prime notion for alternating surface link projections. However, since $F$ has a boundary we have to adjust this definition slightly. For example, if the virtual link has only one component, then the boundary is a 2-cycle in the cell decomposition dual to the projection. We fix this by considering edge cycles that use only one of the boundary edges.

A link projection on a surface $F$ is called alternating if one encounters over- and under-crossings alternately as one travels along any component. Since a 2-cell in the decomposition of $F$ dual to the projection comes from a crossing, two opposing edges in it are connected by the over-crossing. We refer to these edges as the over-crossing edges. The other two opposing edges are referred to as under-crossing edges.

Definition 1.1. Given an alternating link projection on a surface $F$ endowed with the cell decomposition dual to the projection. We say that the arc projection is prime if every reduced edge loop contained in $F^{(1)}-$ \{over-crossing boundary edges\} or in $F^{(1)}-$ \{under-crossing boundary edges $\}$ has length at least four. Furthermore we say the arc projection is dense if every reduced edge loop in $F^{(1)}-$ \{over-crossing boundary edges\} or in $F^{(1)}-$ \{under-crossing boundary edges $\}$ that presents a non-trivial element in the fundamental group of the surface $F$ has length at least five. 
Note that for an alternating link there is a checkerboard coloring to the regions of $F$ determined by the projection. One color corresponds to a cycle of under-crossings as one travels around the boundary of the region (in counterclockwise fashion, say), and the other color corresponds to a cycle of over-crossings. This checkerboard coloring implies that the 1-skeleton of the cell decomposition dual to the projection is a bi-partite graph, and so we just have to rule out 2-cycles to see that the smallest loop is of length at least 4 . So our conditions are not too stringent. This point of view is in D. Wise's thesis [19]. Note that dense implies that there are more than two strands of the projection running across every handle of the surface. It was shown in [5] that prime alternating link projections on surfaces have aspherical Wirtinger presentations. Here is one of the central results of this article.

Theorem 1.2. Given a prime alternating link projection on the surface $F$. Assume that the projection does not contain 2- and 3-tangles (see Figure 1). Then $G$, the group defined by the Wirtinger presentation of the projection, is the fundamental group of a finite piecewise-Euclidian 2-complex of non-positive curvature. Furthermore, if the link projection is dense, then $G$ is hyperbolic.
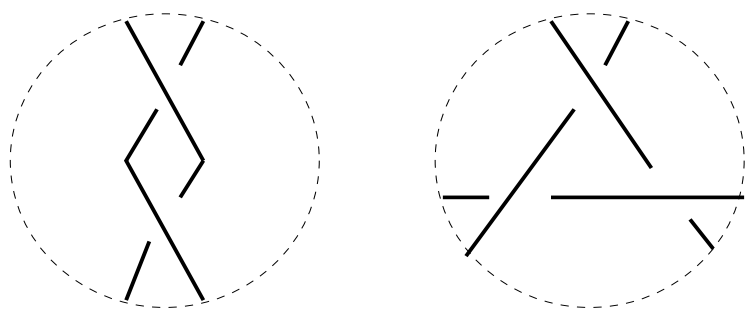

Figure 1. 2- and 3-tangles.

Remark. A link projection on a disc $F$ that has only one component has to contain a 2- or a 3-tangle. Consider the cell decomposition of $F$ dual to the projection. The disc $F$ is tiled by 4 -gons and has two edges in its boundary. Thus, for curvature reasons, there has to be an interior vertex of valency less than four.

In case $F$ is a disc the first part of the theorem (not concerning hyperbolicity) is well known and holds without having to rule out 2- and 3-tangles. In fact the Dehn complex associated with the projection is a non-positively curved square complex with fundamental group $G$. See for example [3], the section on knots. The fact that groups of labeled oriented intervals can be hyperbolic (and hence are not knot groups) was first observed by Rosebrock [16]. Other hyperbolic LOT (labeled oriented tree) groups are in the literature. We will say more about this in the last section when we discuss examples. 


\section{Almost 3-manifolds and their spines}

An almost 3-manifold is a 3-dimensional cell complex where all vertex links are surfaces. In this section we define three almost 3-manifolds associated with an alternating surface link projection, $M, W$ and $D$ and 2-dimensional spines $M_{s} \subseteq M$, $W_{s} \subseteq W$ and $D_{s} \subseteq D$.

2.1. The link complement and its spine. Given an alternating surface link projection on the surface $F$ endowed with the square cell decomposition dual to the link, we can fatten the surface to $F \times[0,1]$ and remove a properly embedded link that maps to the link projection under $F \times[0,1] \rightarrow F \times\{0\}$. If we remove an open neighborhood of the link we arrive at a 3-manifold $M$ whose boundary is a closed orientable surface of genus $2 g+k$, where $g$ is the genus of $F$ and $k$ is the number of components of the link.

We can widen the removed link neighborhood and arrive at a 2-dimensional spine $M_{s}$ of $M$ (see [5]). In the following we give a detailed description of the cell structure of $M_{s}$. The manifold $M$ is built up from cubes, each cube having an over- and undercrossing arc removed. First we collapse the vertical edges $v \times[0,1]$ of $M, v$ being a vertex in $F$, to the corresponding base vertex $v$. We arrive at a cell complex with vertices in one-to-one correspondence to the vertices in $F$. For every edge $e$ in $F$ we have two edges $e^{t}$ and $e^{b}$ in this complex, a top and a bottom edge. Widening the removed arcs leaves one with three square 2-cells for each cube, the bottom 2-cell being a square in $F \times\{0\}$, the top 2-cell being the square in $F \times\{1\}$ and a mixed square coming from the removed arcs with boundary running alternately along top and bottom edges. We denote this 2-complex by $M_{s}$. In summary, a crossing in the surface link projection (corresponding to a 2-cell in the associated Wirtinger complex) defines a square 2-cell of the projection surface, which yields three square 2-cells of the spine $M_{s}$, a top square, a bottom square and a mixed square. This entire process is depicted in Figure 2.

Vertex links in $M_{s}$. Let $v$ vertex in $M_{s}$ that is an interior vertex of $F$. Then the link of $v$ in $M_{S}$ consists of a bottom circle and a top circle coming from the bottom surface $F \times\{0\}$ and the top surface $F \times\{1\}$, respectively. There are also edges in the link connecting link vertices of the bottom circle and the top circle. They come from the corners in the mixed squares. If $\alpha=\{p, q\}$ is a corner in a square of $F$ connecting points $p$ and $q$ on the sides of the square, then we have corners $\alpha^{t}=\left\{p^{t}, q^{t}\right\}, \alpha^{b}=\left\{p^{b}, q^{b}\right\}$ and $\alpha^{m}=\left\{p^{t}, q^{b}\right\}$ (or $\alpha^{m}=\left\{p^{b}, q^{t}\right\}$ ) coming from the corresponding top, bottom and mixed squares. A typical link is shown in Figures 3 and 4 . If $v$ is a boundary vertex of $F$ the situation is similar. Top and bottom circles as shown on the left side in Figure 4 are replaced by top and bottom arcs.

Theorem 2.1. Given a prime alternating link projection on a surface $F$ that does not contain 2- and 3-tangles (see Figure 1). Then the spine $M_{s}$ is a non-positively curved squared complex. Furthermore, $H=\pi_{1}\left(M_{S}\right)$ is hyperbolic. 

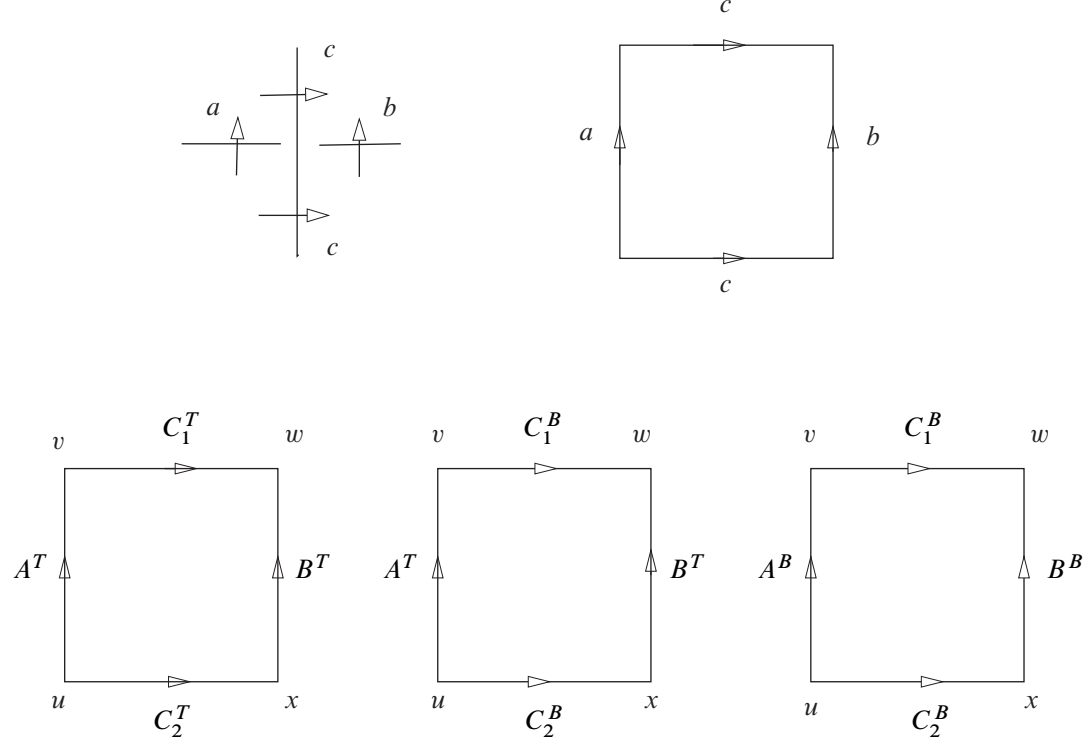

Figure 2. The top row shows a crossing in the link projection and the corresponding 2-cell in the Wirtinger complex. The row below shows the three corresponding 2-cells in $M_{s}$.

Proof. We give each 2-cell in $M_{S}$ the metric of the unit square in the plane. The assumptions that there are no 2- and 3-tangles in the arc projection implies that there are at least four squares grouped around every interior vertex of $F$. It follows that a reduced edge loop in the link of a vertex in $M_{S}$ has length at least four (see Figure 4). Hence $M_{S}$ is non-positively curved (see [3]).

We will next show that the universal covering $\tilde{M}_{S}$ does not contain an isometrically embedded flat plane. Hyperbolicity of the fundamental group of $M_{S}$ will then follow from [3], Theorem 3.1, p. 459. Note first that the projection surface $F$ is a retract of $M_{s}$. We identify $F$ with the top surface in $M_{s}$. A retraction $r: M_{S} \rightarrow F$ is obtained by identifying top and bottom edges that connect the same vertices, and then identifying 2-cells whose boundary edges have already been identified.

Suppose that $E$ is a flat plane in $\tilde{M}_{s}$. If we label the cells in $E$ by their images under the covering projection then $E$ is a Euclidian plane, tiled with the unit squares from $M_{s}$. One should think of $E$ as a Van Kampen diagram where the underlying space is a plane. We call a square in $E$ a $t-, b$-, or $m$-square if it is labeled by a top, bottom or mixed square, respectively. An edge $\tilde{e}$ in $E$ is called a folding edge if the two squares of $E$ that contain $\tilde{e}$ in their boundary are mapped to the same square in $F$ under the composition of the covering map and the retraction $r$. A folding pair are two squares that share a folding edge. Note that a folding pair consists of an $m$-square and either a $t$ - or a $b$-square. A folding line in $E$ is a geodesic that consists entirely of folding edges. 

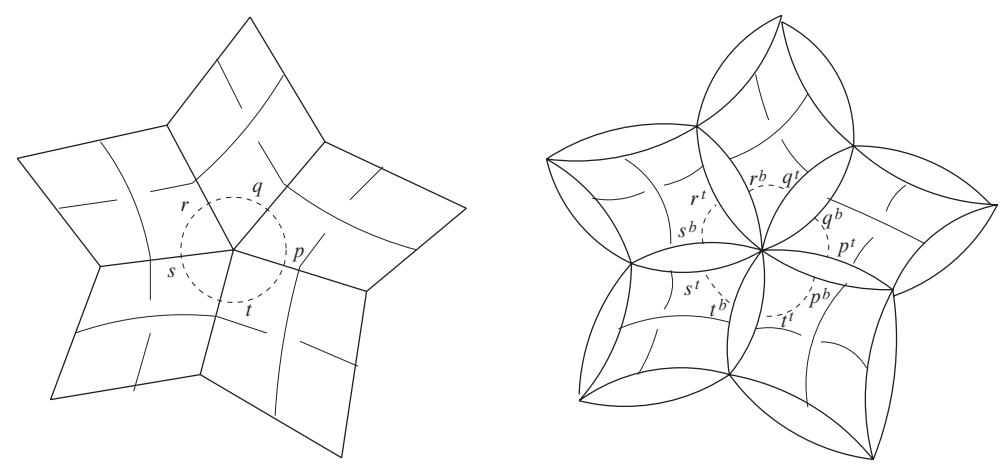

Figure 3. The left side shows the link of a vertex $v$ in the projection surface. Since there is a top and a bottom version of that surface contained in $M_{S}$, the link of $v$ in $M_{S}$ contains a top and a bottom circle. On the right is the part of $M_{S}$ made up by the mixed squares with vertex $v$. These squares contribute edges in the link that connect the top and bottom circle.
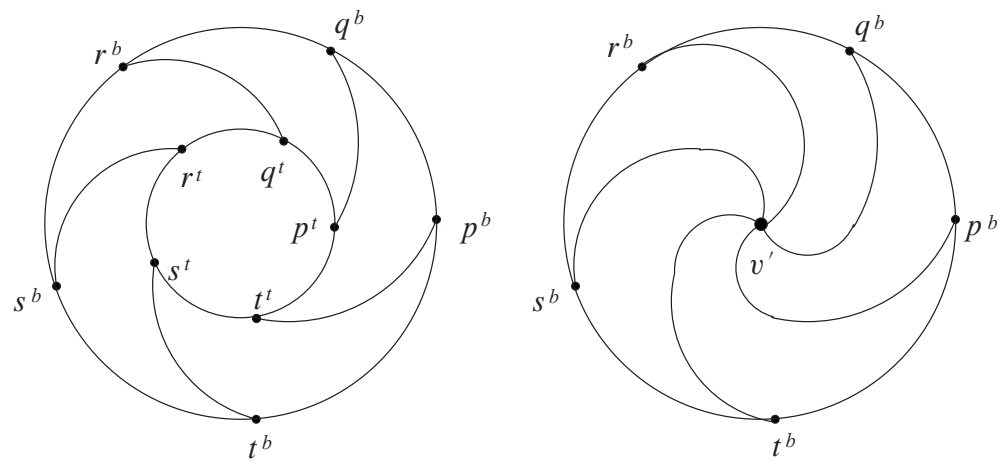

Figure 4. The graph on the left is the link of an interior vertex $v$ in $M_{S}$. It consists of a top and a bottom circle, together with edges connecting these circles that come from corners in mixed squares with vertex $v$. The graph on the right is the link of the same interior vertex in $W_{s}$.

We first argue that $E$ has to contain a folding edge. Note that if we start at a square in $F$ and travel along a gallery of adjacent squares we will end up at the boundary. This is because every such gallery follows a component of the link projection and each such component starts and ends at the boundary. In particular there is no closed gallery in $F$, every gallery starts and ends at the boundary and has length bounded by the number of squares in $F$. Let $\rho$ be the maximal length of a gallery of adjacent squares in $F$. Now if we travel along any gallery of adjacent squares in $E$ for at most $\rho$ squares, we will encounter a folding edge in $E$, because within that distance we encounter an edge that maps to a boundary edge in $F$ under the composition of the covering map and the retraction. 
Next we argue that if $\tilde{e}$ is a folding edge then the geodesic in $E$ that contains that edge is a folding line. Let $\tilde{v}$ be a vertex of the folding edge $\tilde{e}$. Note that one of the four squares grouped around $\tilde{v}$ is an $m$-square because the folding edge $\tilde{e}$ is an edge of an $m$-square. The link $\operatorname{lk}_{E}(\tilde{v})$ of $\tilde{v}$ in $E$ is a reduced cycle of length four in $\operatorname{lk}_{\tilde{M}_{S}}(\tilde{v})=\operatorname{lk}_{M_{S}}(v)$ (the links are identified under the covering map), where $v$ is the image of $\tilde{v}$ under the covering projection. The left side of Figure 4 shows that link. If $v$ is a boundary vertex the top and bottom circles in the link are replaced by arcs. Because one of the squares with vertex $\tilde{v}$ is an $m$-square we may assume without loss of generality that the 4-cycle is of the form $p^{t} q^{t} \cdot q^{t} r^{b} \cdot r^{b} q^{b} \cdot q^{b} p^{t}$ (here $p^{t} q^{t}$ denotes the edge in the link connecting $p^{t}$ to $q^{t}$ ). Under the composition of the covering map and the retraction this cycle maps to $p q \cdot q r \cdot r q \cdot q p$. Hence the $2 \times 2$-segment with center $\tilde{v}$ contains exactly two folding edges, corresponding to vertices $p^{t}$ and $r^{b}$ in $\mathrm{lk}_{E}(\tilde{v})$, and the folding edges are separated by a non-folding edge, that is, they are part of a geodesic in $E$ that passes through $\tilde{v}$. This shows that the two edges $\tilde{e}$ and $\tilde{f}$ of the geodesic containing $\tilde{e}$ emanating from $\tilde{v}$ are both folding edges. Continuing in this fashion we see that the geodesic that passes through $\tilde{v}$ and contains the edge $\tilde{e}$ is a folding line. (In fact developing locally from a folding pair shows that $E$ is tiled in a checker board fashion if we color $m$-squares white and $t$ - and $b$-square black.)

Now consider a bi-infinite gallery of adjacent squares with one boundary a folding line. Starting at some square and traveling along the gallery a distant not greater than $\rho$ we encounter a folding edge that is perpendicular to our folding line. Thus there are perpendicular folding lines in $E$. Let $\tilde{u}$ be the vertex of intersection of the two lines. Then the $2 \times 2$-segment of four squares grouped around $\tilde{u}$ contains perpendicular folding edges. But we have just observed that a $2 \times 2$-segment in $E$ contains exactly two folding edges, and they are part of a geodesic. We have reached a contradiction.

Remark. We are indebted to the referee for providing the combinatorial proof just given. Our original proof was based on Thurston Hyperbolization: If $M$ is a compact, orientable, irreducible and atoroidal 3-manifold which is Haken (i.e. contains a properly embedded incompressible surface) and $\pi_{1}(M)$ is not virtually abelian, then the interior of $M$ admits a complete hyperbolic structure. See [10] for a proof. The manifold $M=F \times I-\{$ knotted link $\}$ is irreducible, orientable and atoroidal in case the knotted link is prime ("atoroidal" being the non-obvious assertion). In the classical setting where $F$ is a disc and the closed knotted link is not a torus knot this was shown by Menasco [13]. His proof can be adopted for the general case. The boundary $\partial M$ is an incompressible surface, so $M$ is Haken. Since $\pi_{1}(\partial M)$ is a subgroup of $\pi_{1}(M)$, and the genus of $\partial M$ is at least two, it follows that $\pi_{1}(M)$ is not virtually abelian. So all the conditions for Thurston Hyperbolization are satisfied. Since $M$ does not have torus boundary components the hyperbolic structure on the interior of $M$ can be taken convex co-compact (see Section 5 of Morgan [14] or Kapovich [10]), which implies that $\pi_{1}(M)$ is $\delta$-hyperbolic. 
2.2. The Wirtinger spine. An almost 3 -manifold $W$ is obtained from the manifold $M$ by coning off the top surface $F \times\{1\}$. We denote the cone point by $T$ and call $W$ the Wirtinger space associated with the surface link projection. It was shown in [5] that $W$ is homotopically equivalent to the Wirtinger complex of the surface link projection. In particular the 2-dimensional spine $W_{s}$ of $W$ constructed below is homotopically equivalent to the Wirtinger complex.

The procedure of collapsing $M$ to $M_{s}$ can be applied to $W$ and we arrive at a 3-complex that contains $M_{s}$, with the top surface coned off. We collapse the 3-cells by pushing in the squares of the top surface. Note that the only 2-cells attached to a top edge are a triangle coming from coning off this edge together with one mixed square in case the edge is an interior edge or an under-crossing boundary edge (recall that we assume the link projection to be alternating). We push every edge across its cone triangle onto the two edges of the triangle that are attached to the cone vertex $T$. This results in a cell structure since, as was pointed out already, a top edge belongs to at most one mixed square. The resulting cell structure is as follows. The vertices are the vertices of the (bottom) surface $F$ together with the cone vertex $T$, we have the edges from the bottom surface together with edges $\hat{v}$ connecting a vertex $v$ of $F$ to the cone vertex $T$. The 2-cells are the squares from the bottom surface together with hexagonal 2-cells coming from the mixed squares in $M_{s}$ with top edges coned off. See Figure 5.

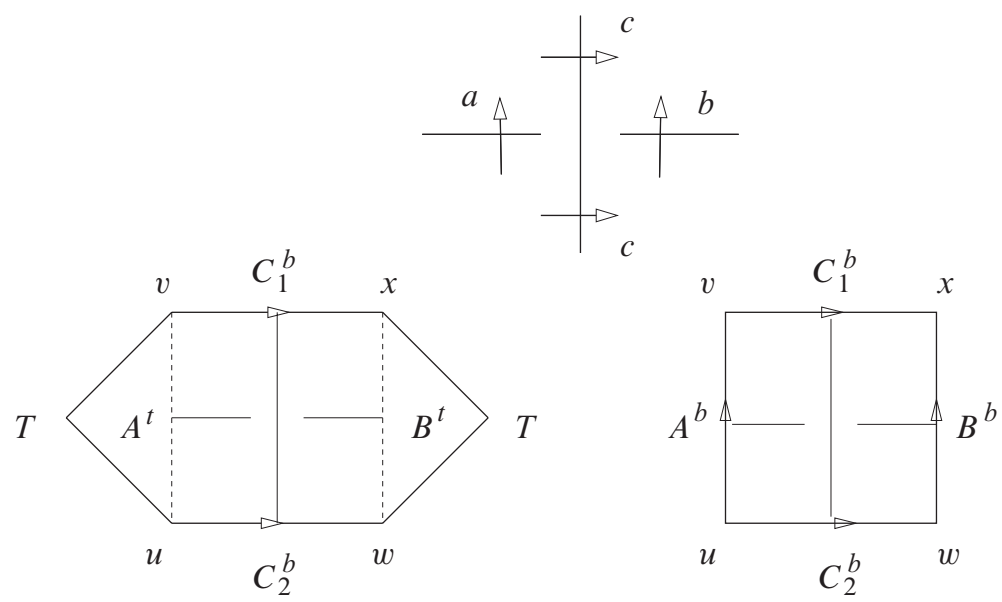

Figure 5. A crossing in the link projection yields two 2-cells in $W_{s}$, a hexagon and a bottom surface square.

Vertex links in $W_{s}$. The spine $W_{s}$ is obtained from $M_{s}$ by coning off the top surface and then collapsing the 3-cells across the surface squares. Every interior top edge is now the boundary of exactly two 2-cells, the cone triangle for that edge and a mixed square (recall that the surface link projection is assumed to be alternating). 
We produced a hexagonal 2-cell by internally collapsing the cone triangle across the top edge. The top edge remains visible in the link of the top vertex $T$. See Figure 5 . A top edge $e^{t}$ in the boundary of $F$ is contained in a mixed square only if it is an under-crossing edge (see the paragraph before Definition 1.1 for the definition of over- and under-crossing edges). If $e^{t}$ does not belong to any mixed square then this edge will disappear when the cone triangle is collapsed. This shows that the link of $T$ in $W_{s}$ is $F^{(1)}-$ \{over-crossing boundary edges $\}$. The link of an interior vertex $v$ in $W_{s}$ is obtained from the link of $v$ in $M_{s}$ by coning off the top circle, then collapsing the triangles by pushing in the edges of the top circle. Vertices of the top circle all have valency two and can be left off. See the left side of Figure 4. If the vertex $v$ is a boundary vertex, then the situation is similar, the circle is replaced by an interval.

2.3. The Dehn spine. An almost 3-manifold $D$ is obtained from $W$ by coning off the bottom surface $F \times\{0\}$. We denote the cone point by $B$ and call $D$ the Dehn space associated with the surface link projection. A 2-dimensional spine $D_{s}$ is obtained by a procedure analogues to the one given in the previous subsection, 3-cells are collapsed across the bottom surface squares. We now collapse the triangle which is the cone on the boundary edge $f$ and perform internal collapses as before. The result is a 2-complex with two vertices, $\mathrm{T}$ and $\mathrm{B}$, edges in one-to-one correspondence with the vertices in $F$ and one square 2-cell for every crossing in the projection. See Figure 6 . The link of $T$ in $D_{s}$ is $F^{(1)}-$ \{over-crossing boundary edges $\}$ and the link of $B$ is $F^{(1)}-$ \{under-cossing boundary edges $\}$. In [5] we used this spine to prove that prime alternating surface link projections have aspherical Wirtinger complexes.
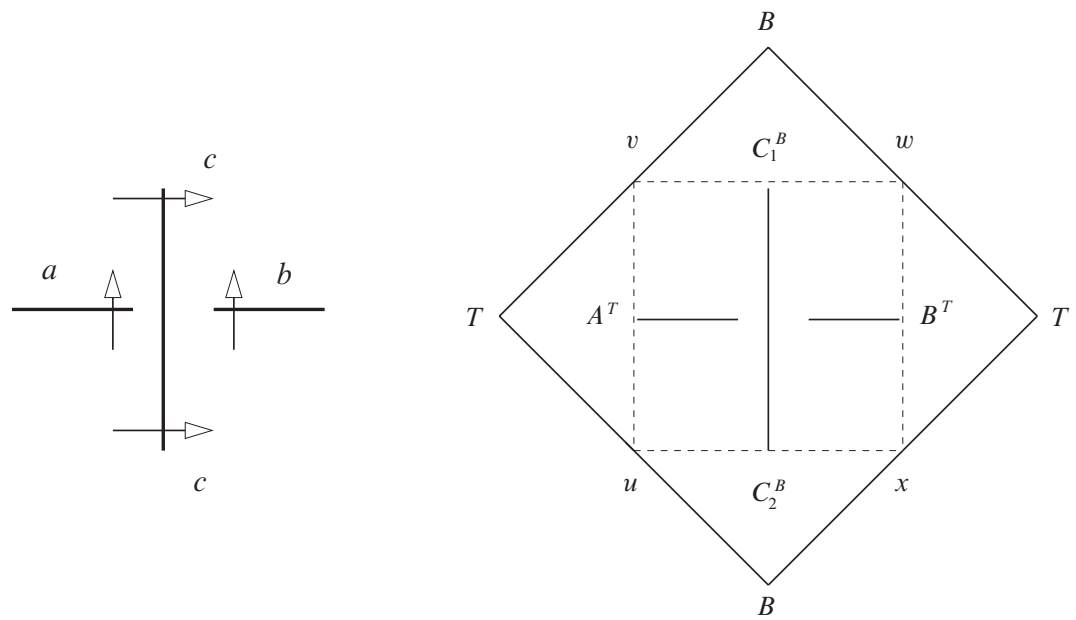

Figure 6. A crossing in the link projection gives a square 2-cell in $D_{s}$. 


\section{The geometry of the Wirtinger spine}

Theorem 3.1. Given a prime alternating link projection on a surface $F$ that does not contain 2-or 3-tangles (see Figure 1). Then the 2-complex $W_{s}$ can be made into a non-positively curved piecewise-Euclidian complex. If we further assume that the projection is dense then $G=\pi_{1}\left(W_{s}\right)$ (which is isomorphic to the group defined by the Wirtinger presentation of the link projection) is hyperbolic.

Proof. We give each square 2-cell in $W_{s}$ the metric of the unit square in the plane, in particular all corner angles are $\pi / 2$. Each hexagonal 2-cell is metrized as a planar hexagon, the angle at the two $T$-vertices being $\pi / 2$, the four remaining angles being $3 \pi / 4$. Alternating implies that the link of $T$ in $W_{s}$ is $F^{(1)}-$ \{over-crossing boundary edges\}. Prime implies that there are no reduced edge loops of length less that four in $F^{(1)}-$ \{over-crossing boundary edges\}, so each edge loop in the link has length at least $2 \pi$. Next consider the link of a vertex $v$. In case $v$ is interior, this link is a circle with vertices coned off (see the right side of Figure 4). Since the arc projection does not contain 2-or 3-tangles, this circle contains at least four edges. Thus a reduced edge loop in the link that is part of the circle contains at least four circle edges and hence has length at least $2 \pi$. A reduced edge loop in the link that contains a cone edge (an edge not part of the circle) contains an even number of cone edges, each of length $3 \pi / 4$, and at least one circle edge and hence also has length at least $2 \pi$. If $v$ is a boundary vertex the situation is similar, the circle is replaced by an interval. We see that there are no loops shorter that $2 \pi$ in the vertex links and hence $W_{S}$ is indeed non-positively curved [3].

Now let us assume that the surface link projection is dense and either $F$ is a disc and the link projection has at least two components, or the genus of $F$ is at least two. In order to show that $G$ is hyperbolic we need to prove that the universal covering $\widetilde{W}_{s}$ does not contain flat planes (see [3], Theorem 3.1, p. 459). Suppose there is a flat $E$ in $\widetilde{W}_{s}$. Then $E$ is tessellated with the metrized 2-cells of $W_{s}$, that is with the flat squares and hexagons of $W_{S}$ (see Figure 7). The vertex links in this tessellation are reduced edge loops in vertex links of $W_{s}$ of length $2 \pi$. If the tessellation contains a vertex labeled $T$, then there are four hexagons placed around that vertex and the link is an edge loop of length four in the 1-skeleton of the top surface. Denseness implies that this edge loop represents the trivial element in $\pi_{1}(F)$. In fact, the edge loop bounds a 2 -cell in the top surface. This can be seen as follows. Since the loop is homotopically trivial, we can built a reduced Van Kampen disc tessellated with the square 2-cells of $F$ whose boundary is the edge loop of length four under consideration. Since $F$ itself is a non-positively curved squared complex the disc is as well. All squares in the Van Kampen disc have curvature zero and the curvature at an interior vertex is less or equal to zero. It follows from the combinatorial Gauss-Bonnet formula (see for example McCammond[12], page 41) that the curvature at each boundary vertex is $\pi / 2$, so the Van Kampen disc consists of a single square. We can cut off the four triangles around the $T$-vertex and replace it by that square 2-cell of the top 


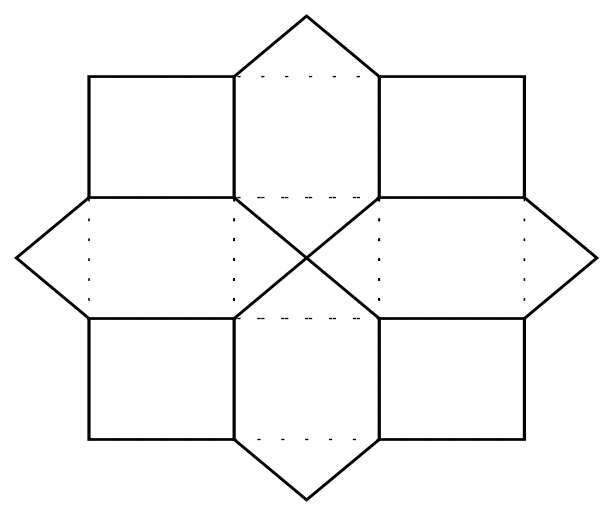

Figure 7. A tessellation of the plane with the metric 2-cells of $W_{s}$. The dotted lines indicate top edges. These edges are present in $W$ (but not in the spine $W_{S}$ ). The link at the center vertex is a reduced edge loop of length four in the 1-skeleton of the top surface, hence it is the boundary of a 2-cell of the top surface (which is part of $M_{S}$ ).

surface. In this way we built tessellation of the Euclidian plane by square 2-cells of $M_{s}$ and each vertex link in this tessellation is a reduced edge loop in some vertex link of $M_{S}$ of length $2 \pi$. Topologically phrased, we pull the flat plane $E \subseteq \widetilde{W}_{S} \subseteq \widetilde{W}$ off $p^{-1}(T)$, where $p: \tilde{W} \rightarrow W$ is the covering projection, and obtain a flat plane $\bar{E} \subseteq \bar{M}_{s}$, where $\bar{M}_{s}$ is an intermediate covering of $M_{s}$ (recall that $M_{S} \subseteq M$ is contained in $W$.) That flat plane lifts to a flat plane in $\tilde{M}_{s}$. This contradicts the fact that, since $H=\pi_{1}\left(M_{S}\right)$ is hyperbolic by Theorem 2.1 , there are no flats in $\tilde{M}_{S}$ (see [3], Theorem 3.1, p. 459).

An analog result holds for the Dehn complex.

Theorem 3.2. Given a prime alternating link projection on a surface $F$. Then the 2-complex $D_{s}$ is a non-positively curved squared complex. If we further assume that the link projection does not contain 2- or 3-tangles and the projection is dense, then $Q$ is hyperbolic.

Proof. We give each 2-cell in $D_{s}$ the metric of the unit square in the plane, in particular all corner angles are $\pi / 2$. Alternating says that the links of $T$ and $B$ are $F^{(1)}-$ \{over-crossing boundary edges\} and $F^{(1)}-$ under-crosdding boundary edges\}, respectively, and prime says that the shortest reduced edge loop in these links has length at least four. This implies that $D_{s}$ is non-positively curved. Dense implies that there are no flats in the universal covering of $D_{s}$. This can be seen by the same arguments as given in the proof of Theorem 3.1. 


\section{Examples}

We use the results from the previous sections to construct virtual link groups that are CAT(0), that is fundamental groups of non-positively curved piecewise-Euclidian complex, and hyperbolic. Other examples are in the literature, the first ones are Rosebrock's [16]. The paper of Brady-Barnard contains LOT groups that are CAT(0) and hyperbolic and free-by-cyclic.

The simplest example that fits our conditions is the following:

Example 4.1. Consider a link projection on a disc, one component running west to east, the other running north to south, over-crossing the west-east component.

Clearly, this projection does not contain 2- or 3-tangles and is prime. In fact, $F$ consists of a single square. Thus $G=\pi_{1}(M)=\pi_{1}(W)=\pi_{1}(D)$ is hyperbolic. The Wirtinger presentation of the projection is $\langle a, b, c \mid a c=c b\rangle$, so $G$ is free of rank 2.

Example 4.2. Consider the square $[0, n] \times[0, n], n$ an odd number in $\mathbb{N}$. Draw lines from $(0, j)$ to $(n, j+1), j=0, \ldots, n-1$ and also from $(i, n)$ to $(i+1,0)$, $i=0, \ldots, n-1$. Starting at $(0,0)$, travel along these lines from left to right, make the first crossing an over-crossing, the next an under-crossing and so on. Now cut off the four corners and identify opposite sides. We obtain an alternating link projection $A(n)$ with two components on a punctured torus. See Figure 8.
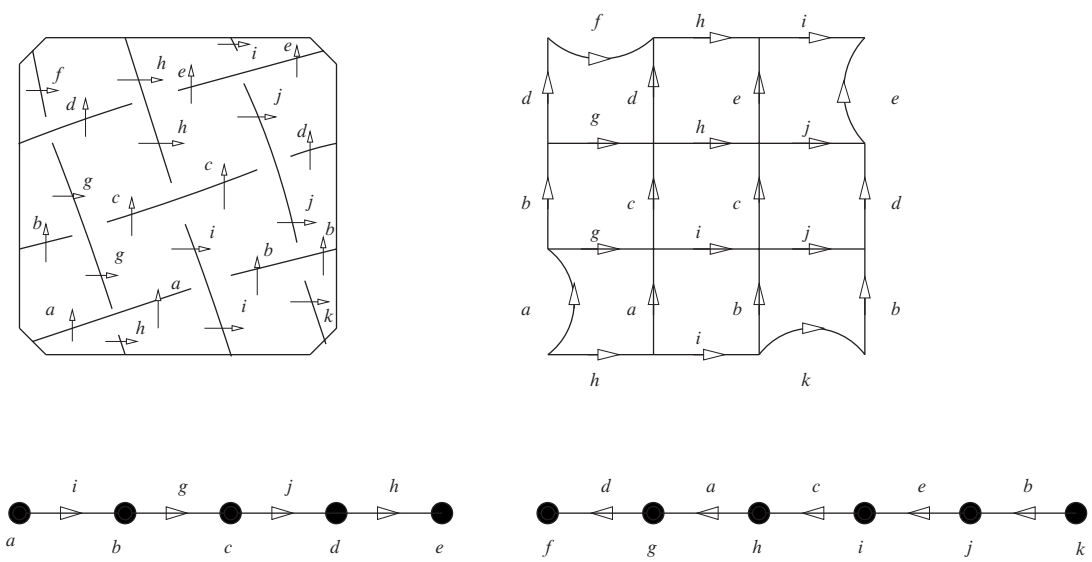

Figure 8 . The left side shows the surface link projection $A(3)$. On the right we see the projection surface, a punctured torus, endowed with the square cell decomposition dual to the link projection. Boundary edges that carry the same label are to be identified.

Note that $A(n)$ does not contain 2- or 3-tangles. The fact that $n$ is odd ensures that each component of the link is alternating and for $n \geq 5$ the projection is dense. The 
shortest edge loop in $F^{(1)}-$ the two over-crossing boundary edges $\}$ and in $F^{(1)}-$ \{the two under-crossing boundary edges\} ( $F$ being the projection surface, i.e., the punctured torus), that does not represent the trivial element of $\pi_{1}(F)$ has length $n+1$. Our theorems implies that all groups $\pi_{1}(M), \pi_{1}(W)$ and $\pi_{1}(D)$ are CAT $(0)$ and hyperbolic.

\section{References}

[1] J. Barnard and N. Brady, Distortion of surface groups in CAT(0) free-by-cyclic groups. Geom. Dedicata 120 (2006), 119-139. Zbl 1167.20024 MR 2252898

[2] M. Bestvina and N. Brady, Morse theory and finiteness properties of groups. Invent. Math. 129 (1997), 445-470. Zbl 0888.20021 MR 1465330

[3] M. R. Bridson and A. Haefliger, Metric spaces of non-positive curvature. Grundlehren Math. Wiss. 319, Springer-Verlag, Berlin 1999. Zbl 0988.53001 MR 1744486

[4] W. A. Bogley, J.H.C. Whitehead's asphericity question. In Two-dimensional homotopy and combinatorial group theory, London Math. Soc. Lecture Note Ser. 197, Cambridge University Press, Cambridge 1993, 309-334. Zbl 0811.57008 MR 1279184

[5] J. Harlander and S. Rosebrock, Generalized knot complements and some aspherical ribbon disc complements. J. Knot Theory Ramifications 12 (2003), 947-962. Zbl 1053.57005 MR 2017964

[6] J. Howie, Some remarks on a problem of J. H. C. Whitehead. Topology 22 (1983), 475-485. Zbl 0524.57002 MR 715251

[7] J. Howie, On the asphericity of ribbon disc complements. Trans. Amer. Math. Soc. 289 (1985), 281-302. Zbl 0572.57001 MR 779064

[8] G. Huck and S. Rosebrock, Aspherical labelled oriented trees and knots. Proc. Edinb. Math. Soc. (2) 44 (2001), 285-294. Zbl 0983.57003 MR 1880392 (2002k:57004)

[9] G. Huck and S. Rosebrock, Spherical diagrams and labelled oriented trees. Proc. Roy. Soc. Edinburgh Sect. A 137 (2007), 519-530. Zbl 1134.57002 MR 2332680

[10] M. Kapovich, Hyperbolic manifolds and discrete groups. Progr. Math. 183, Birkhäuser, Boston 2001. Zbl 0958.57001 MR 1792613

[11] L. H. Kauffman, Virtual knot theory. European J. Combin. 20 (1999), 663-690. Zbl 0938.57006 MR 1721925

[12] J. McCammond, Constructing non-positively curved spaces. In Geometric and cohomological methods in group theory, London Math. Soc. Lecture Note Ser. 358, Cambridge University Press, Cambridge 2009, 162-224. Zbl 05859856 MR 2605177

[13] W. Menasco, Closed incompressible surfaces in alternating knot and link complements. Topology 23 (1984), 37-44. Zbl 0525.57003 MR 721450

[14] J. W. Morgan, On Thurston's uniformization theorem for three-dimensional manifolds. In The Smith conjecture, Pure Appl. Math. 112, Academic Press, Orlando 1984, 37-125. Zbl 0599.57002 MR 758464 
[15] D. Rolfsen, Knots and links. Math. Lecture Ser. 7, Publish or Perish, Berkeley 1976. Zbl 0339.55004 MR 0515288

[16] S. Rosebrock, On the realization of Wirtinger presentations as knot groups. J. Knot Theory Ramifications 3 (1994), 211-222. Zbl 0824.57005 MR 1279923

[17] S. Rosebrock, The Whitehead conjecture - an overview. Sib. Èlektron. Mat. Izv. 4 (2007), 440-449. MR 2465444 http://semr.math.nsc.ru/v4.html

[18] C. M. Weinbaum, The word and conjugacy problems for the knot group of any tame, prime, alternating knot. Proc. Amer. Math. Soc. 30 (1971), 22-26. Zbl 0228.55004 MR 0279169

[19] D. T. Wise, Non-positively curved squared complexes, aperiodic tilings, and nonresidually finite groups. Ph.D. thesis, Princeton University, Princeton 1996.

http://www.math.mcgill.ca/wise/papers.html

Received July 12, 2010; revised October 26, 2010

J. Harlander, Department of Mathematics, Boise State University, 1910 University Drive, Boise, ID 83725-1555, U.S.A.

E-mail: jensharlander@boisestate.edu 